\title{
New paradigms on hematopoietic stem cell differentiation
}

\author{
Hui Cheng ${ }^{1,2,3,4 \bowtie}$, Zhaofeng Zheng ${ }^{1,2,4}$, Tao Cheng ${ }^{1,2,3,4}$ \\ ${ }^{1}$ State Key Laboratory of Experimental Hematology, Chinese Academy of Medical Sciences and Peking Union Medical \\ College, Tianjin 300020, China \\ 2 Institute of Hematology and Blood Disease Hospital, Chinese Academy of Medical Sciences and Peking Union Medical \\ College, Tianjin, China \\ ${ }^{3}$ Center for Stem Cell Medicine, Chinese Academy of Medical Sciences, Tianjin, China \\ ${ }^{4}$ Department of Stem Cell and Regenerative Medicine, Peking Union Medical College, Tianjin, China \\ $\triangle$ Correspondence: chenghui@ihcams.ac.cn (H. Cheng), chengtao@ihcams.ac.cn (T. Cheng) \\ Received April 2, 2019 Accepted April 25, 2019
}

\begin{abstract}
Ever since hematopoietic stem cells (HSCs) were first identified half a century ago, their differentiation roadmap has been extensively studied. The classical model of hematopoiesis has long held as a dogma that HSCs reside at the top of a hierarchy in which HSCs possess self-renewal capacity and can progressively give rise to all blood lineage cells. However, over the past several years, with advances in single cell technologies, this developmental scheme has been challenged. In this review, we discuss the evidence supporting heterogeneity within HSC and progenitor populations as well as the hierarchical models revised by novel approaches mainly in mouse system. These evolving views provide further understanding of hematopoiesis and highlight the complexity of hematopoietic differentiation.
\end{abstract}

KEYWORDS hematopoietic stem cell, hierarchy, heterogeneity, differentiation

\section{THE CLASSICAL AND BALANCED HEMATOPOIETIC HIERARCHICAL MODEL}

The cellular potential of hematopoietic stem cells (HSCs) has been traditionally defined by transplanting donor cells (or a single cell) into recipients that are preconditioned by lethal irradiation and therefore devoid of a functional endogenous

Hui Cheng and Zhaofeng Zheng contributed equally to this work. hematopoietic system. This assay has long been the goldstandard for functional HSCs.

The first in vivo evidence for the existence of HSCs, in 1961 , was based on the rescue of lethally irradiated recipient mice by bone marrow transplantation, followed by observing hematopoietic colonies in the spleens of recipients (Till and Mc, 1961). Thereafter, scientists were interested in developing methods to purify HSCs from bone marrow to better understand their function and molecular regulatory networks. Separation of HSCs became possible with the utilization of antibodies and fluorescence-activated cell sorting (FACS). Weissman and colleagues first described HSC-enriched cells using the combination of several surface markers in 1988 (Spangrude et al., 1988). Since then, different groups have put great effort into identifying more surface markers to further purify HSCs. To date, CD34, Sca-1, c-Kit, the signaling lymphocyte activation molecule (SLAM) markers, etc. are still commonly used to isolate HSCs in different labs (Ikuta and Weissman, 1992; Okada et al., 1992; Osawa et al., 1996; Kiel et al., 2005; Oguro et al., 2013). Since similar approaches can be used to identify multi- and unipotent progenitors, different progenitor populations were also isolated based on surface markers (Kondo et al., 1997; Akashi et al., 2000; Adolfsson et al., 2005; Wilson et al., 2008; Pietras et al., 2015).

Through transplantation and colony assay, HSCs have been defined on the basis of two essential properties, selfrenewal and multipotent differentiation, which can produce cells of all blood lineages (Morrison et al., 1995; Orkin, 2000; Reya et al., 2001; Dick, 2003; Reya, 2003). By contrast, progenitors have been defined by the absence of self-renewal and restricted lineage differentiation capacities. To 
better illustrate the relationship between an HSC and its progenies, and the stepwise differentiation process, the immunophenotype-based tree-like hierarchy model was largely established by Weissman's group (Kondo et al., 1997; Morrison et al., 1997; Akashi et al., 2000; Manz et al., 2002). In this classical model, HSCs can be divided into two subpopulations according to their CD34 expression: CD34long-term (LT)-HSCs and CD34 ${ }^{+}$short-term (ST)-HSCs. LTHSCs are a rare, quiescent population in bone marrow and have full long-term (> 3 4 months) reconstitution capacity, whereas ST-HSCs only have a short-term (mostly $<1$ month) reconstitution ability. LT-HSCs differentiate into STHSCs, and subsequently, ST-HSCs differentiate into multipotent progenitors (MPPs), which have no detectable selfrenewal ability (Yang et al., 2005). The first bifurcation occurs between the common myeloid progenitors (CMPs, with myeloid, erythroid and megakaryocytic potential) and common lymphoid progenitors (CLPs, with only lymphoid potential), which are derived from MPPs. The second branch point at CMPs segregates bipotent granulocyte-macrophage (GMPs) and megakaryocyte-erythrocyte progenitors (MEPs). CLPs further form T, B, NK and dendritic cells, while GMPs differentiate into granulocytes/monocytes and MEPs generate megakaryocytes/erythrocytes. All these populations form a tree-like and balanced hierarchy model, within which key transcription factors (TFs) and cytokines precisely conduct the stepwise differentiation of HSCs to mature blood cells (Zhu and Emerson, 2002; Robb, 2007; Metcalf, 2008; Zhang and Lodish, 2008; Seita and Weissman, 2010) (Fig. 1).

\section{ADVANCES IN THE HEMATOPOIETIC HIERARCHY}

Although the classical model has been very useful for understanding the differentiation process of HSCs, it is worth noting that this model has some shortcomings in that it oversimplifies the complexity of hematopoietic stem and progenitor cells (HSPCs), and it is only based on the surface markers and transplantation using bulk cells. Bulk cell analysis assumes that each cell, which has the same phenotype, possesses an identical function. With advances in single cell technology and genetic mouse models, this classical model has been challenged over the past several years, especially in the elucidation of megakaryopoiesis. Moreover, new types of HSPCs have been identified and extensively studied due to their lineage biases.

\section{Heterogeneity in HSCs lineage output and debates on megakaryocyte differentiation}

By using limiting-dilution analysis and single-cell transplantation, the Sieburg and Eaves groups defined myeloid-biased $(\mathrm{My}-\mathrm{Bi})$, balanced $(\mathrm{Ba})$ and lymphoid-biased (Ly-Bi) HSCs based on the ratio of myeloid to lymphoid cells outputs (Muller-Sieburg et al., 2002; Muller-Sieburg et al., 2004; Dykstra et al., 2007; Benz et al., 2012) (Fig. 2A and 2B). In addition, platelet-biased HSCs have also been reported as a My-Bi subset residing at the top of the hematopoietic hierarchy (Sanjuan-Pla et al., 2013) (Fig. 2C). Researchers have long recognized the concept of LT-HSCs and ST-HSCs. Based on the reconstitution time period, intermediate-term HSCs (IT-HSCs), which sit in-between LT-HSC and ST-HSC and contribute to reconstitution up to 8 months after transplantation, have been used in several labs (Benveniste et al., 2010; Yamamoto et al., 2013). In addition, Lu et al. tracked single HSCs in vivo using viral genetic barcoding combined with high-throughput sequencing (Lu et al., 2011). They also revealed heterogeneity in the HSC population. In this assay, they showed that that HSCs do not equally contribute to progenies, and that two distinct HSC differentiation patterns co-exist in the same recipient mouse after irradiation. One differentiation pattern consists of progenitor cell populations including GMPs, MEPs and CLPs; the other group consists of mature lymphoid blood cells. Similarly, with single cell transplantation, Yamamoto et al. observed that self-renewing lineage-restricted progenitors exist in phenotypically defined HSC, containing megakaryocyte repopulating progenitors (MkRPs), megakaryocyte-erythrocyte repopulating progenitors (MERPs), and common myeloid repopulating progenitors (CMRPs) (Yamamoto et al., 2013) (Fig. 2D). This study suggests that oligo-, bi- and unipotent cells co-exist in HSC populations. Furthermore, SLAM family markers CD150 and CD229 can segregate HSCs into different fractions with differentiation reconstitution ability. Compared with CD150 med HSC, CD150 ${ }^{\text {hi }}$ HSC displayed higher self-renewal potential with myeloid biased differentiation (Morita et al., 2010). CD229- HSCs have long-term self-renewal potential with myeloid biased potential and form all of the other stem and progenitor cell populations, whereas CD229 ${ }^{+}$HSCs appear to have less self-renewal capacity with lymphoid biased potential (Oguro et al., 2013). The single-cell omics analyses (Moignard et al., 2013; Wilson et al., 2015; Nestorowa et al., 2016; Buenrostro et al., 2018; Laurenti and Gottgens, 2018; Jacobsen and Nerlov, 2019), including single-cell RNA sequencing (scRNA-seq) and single cell assay for transposase-accessible chromatin using sequencing (scATACseq), have further uncovered the presence of heterogeneity in the most primitive HSC populations.

The origin of megakaryocytes has been under debate for several years. The Jacobsen group identified lymphoidprimed MPPs (LMPPs) that give rise to granulocyte/macrophage and lymphoid lineages but not a megakaryocyte/ erythrocyte lineage (Adolfsson et al., 2005) (Fig. 2C). However, lineage tracing studies challenge this view and suggest that LMPPs also differentiate into a megakaryocyte/erythrocyte lineage (Forsberg et al., 2006; Boyer et al., 2011). The different outcomes may be attributed to the cell dose used for transplantation and the methods, including mouse models, used in different labs. More recently, mRNA expression of the megakaryocyte marker von Willebrand factor (VWF) and the surface receptor c-Kit were suggested to be indicative of a platelet-biased, but 


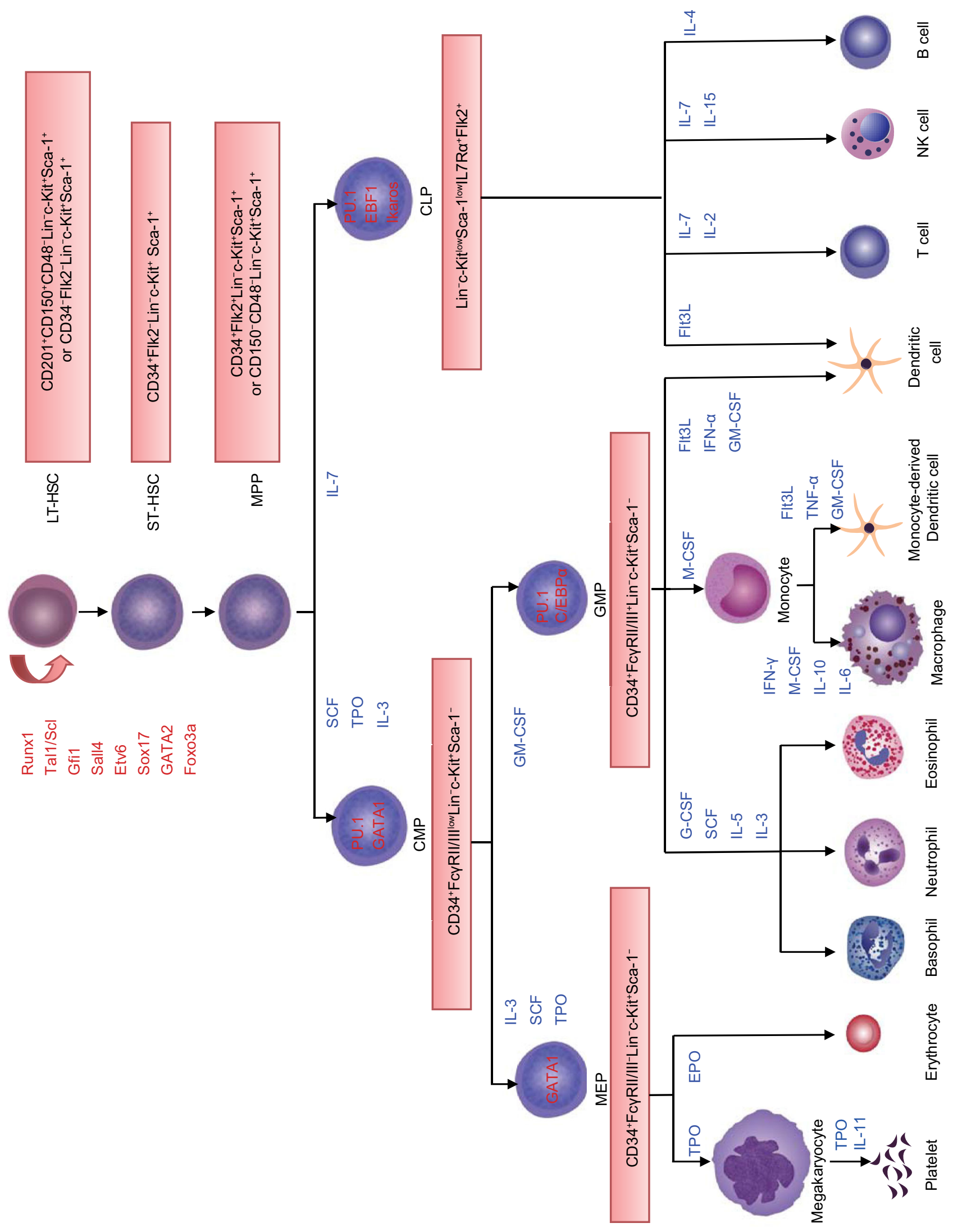


Figure 1. The classical hematopoietic hierarchy. In the classical model, LT-HSCs sit at the top of hierarchy. LT-HSCs differentiate into ST-HSCs, and subsequently to MPPs with reduced self-renewal ability. Downstream of MPPs, a strict separation between the myeloid (CMPs) and lymphoid (CLPs) branches is the first step in lineage commitment. CMPs can generate MEPs and GMPs. CLPs give rise to lymphocytes and dendritic cells. MEPs differentiate into megakaryocytes/platelets and erythrocytes. GMPs produce granulocytes, macrophages, and dendritic cells. Hematopoietic differentiation is controlled by extrinsic cytokines and intrinsic transcription factors.

multipotent HSC sub-population (Sanjuan-Pla et al., 2013; Shin et al., 2014). Evidence for a platelet-biased population of HSCs was provided by the Jacobsen group (Sanjuan-Pla et al., 2013). They found that $25 \%$ of LT-HSCs express vWF and $\mathrm{VWF}^{+} \mathrm{HSCs}$ are primed for platelet-specific gene expression, with enhanced propensity for long-term reconstitution of platelets. The $\mathrm{vWF}^{+}$platelet-primed HSCs also have a long-term myeloid lineage bias, can self-renew, and can give rise to $\mathrm{VWF}^{-}$lymphoid-biased HSCs (Fig. 2C). Therefore, the platelet-primed HSCs sit at the top of the hematopoietic hierarchy. Moreover, based on single-cell transplantation experiments, the existence of megakaryocyte-lineage restricted cells in the phenotypic HSC compartment was proposed (Yamamoto et al., 2013). Pairdaughter cell transplantation assay indicates that megakaryocyte precursors are directly derived from HSCs (Yamamoto et al., 2013) (Fig. 2D). Later, a study reported that the HSC compartment contains stem-like megakaryocyte committed progenitors (SL-MkPs), a cell population that shares many features with HSCs (Haas et al., 2015). This population becomes activated upon inflammatory stress to efficiently replenish platelets, thus a potential shortcut from HSCs to megakaryocytes has been suggested under inflammatory conditions (Haas et al., 2015). Furthermore, by tracking progenitors and mature lineage cells produced from single transplanted HSCs, a recent report from Jacobsen's lab showed that a distinct class of HSCs adopts a fate towards long-term and effective replenishment of megakaryocytes/platelets without replenishment of any other blood cell lineages, whereas no HSCs contribute exclusively to any other single blood cell lineage (Carrelha et al., 2018).

Collectively, HSCs and megakaryocytes share several features, for example, expression of thrombopoietin receptor (MPL), CD150, CXCR4 and vWF, etc. (Wang et al., 1998; Sugiyama et al., 2006; Pronk et al., 2007; Yoshihara et al., 2007; Huang and Cantor, 2009). More importantly, megakaryocytes also serve as an HSC niche component and tightly regulate the maintenance of HSC function (Bruns et al., 2014; Zhao et al., 2014). Platelet- and myeloid-biased VWF$^{+}$HSCs, but not lymphoid-biased VWF $^{-}$HSCs, associate with megakaryocytes and are regulated by megakaryocytes (Pinho et al., 2018). All the evidence suggests that
HSCs and the megakaryocyte (or its progenitors) are closer to one another in the hematopoietic developmental hierarchy than previously appreciated. In consideration of the heterogeneity observed in the HSC population, our view is that lineage (cell fate) predetermination occurs in HSCs, prior to their differentiation towards progenitors. Moreover, the megakaryocyte can arise independent of other lineages, and the megakaryocyte differentiation route is first separated from other blood cell lineages in the hierarchy.

\section{Heterogeneity in MPPs}

The Trumpp (Wilson et al., 2008) and Passegue (Pietras et al., 2015) groups further divided the MPP population into MPP1, MPP2, MPP3 and MPP4 according to their immunophenotype, cell cycle status, lineage bias, resistance to drug treatment and bone marrow abundance. MPP1 is more similar to the previously defined IT-HSC or ST-HSC, which have multiple-lineage reconstitution ability up to 4 months in the first transplantation, whereas MPP2/3/4 are devoid of self-renewal potential and only exhibit short-term myeloid reconstitution ability (<1 month). More importantly, MPP2 and MPP3 produce low levels of T and B cells and MPP4 generates low levels of myeloid cells in vivo. In addition, compared with MPP3 and MPP4, MPP2 produces higher levels of platelets. Taken together, MPP2 is a megakaryocyte-biased MPP subset and MPP3 is a myeloid-biased MPP subset. Both MPP2 and MPP3 are functionally distinct from the lymphoid-primed MPP4. HSCs independently generate all three types of lineage-biased MPPs (MPP2-4), but among them, no MPPs are able to generate other MPPs in vivo (Fig. 2E). After transplantation, HSCs first produce myeloid-biased MPPs (MPP1/2) to quickly establish myeloid output, followed by the lymphoid-primed MPP4 subpopulation to rebuild the lymphoid compartment. Therefore, MPPs are a heterogeneous population with different lineage-biased potential both at the cellular and molecular levels.

\section{Heterogeneity and hierarchy within myeloid progenitors}

In the classical model, CMPs and MEPs are separated according to CD34 expression. In the $\mathrm{Lin}^{-} \mathrm{CKit}^{+} \mathrm{Sca} 1^{-}\left(\mathrm{LKS}^{-}\right)$ population, CMPs are CD34 ${ }^{+} \mathrm{CD} 16 / 32^{-}$, whereas MEPs are CD34 ${ }^{-} \mathrm{CD} 16 / 32^{-}$. CMPs are thought to possess oligo-potency, including granulocyte, macrophage, megakaryocyte and erythrocyte differentiation potential. However, CMPs have a low clonal frequency of mixed myeloid colonies, and MEPs also possess a low level of megakaryocyte potential (Nakorn et al., 2003). Therefore, it prompts us to know whether each CMP or MEP has a different lineage potential at the single cell level, i.e., whether every CMP is indeed oligo-potent, and whether MEP is bipotent.

To understand the heterogeneity and lineage commitment in the $\mathrm{LKS}^{-}$myeloid progenitor population, especially in CMPs, Pronk et al. (Pronk et al., 2007) used CD150, CD105 (Endoglin), CD41 and CD16/32 to re-segregate the LKS $^{-}$ 
A

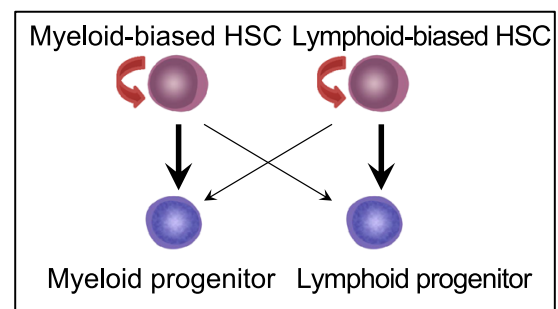

B

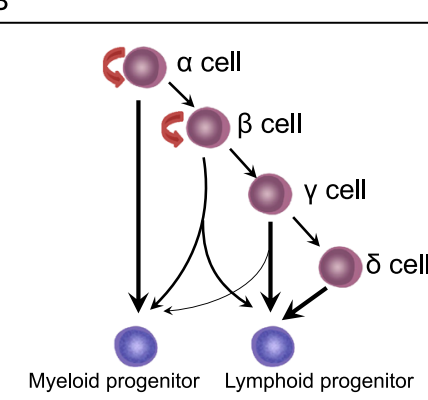

D

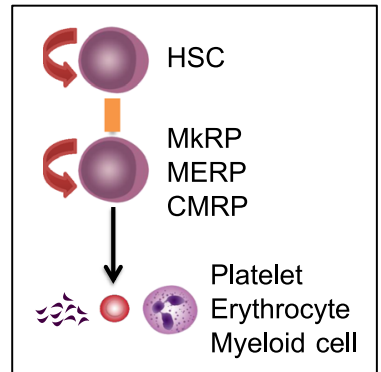

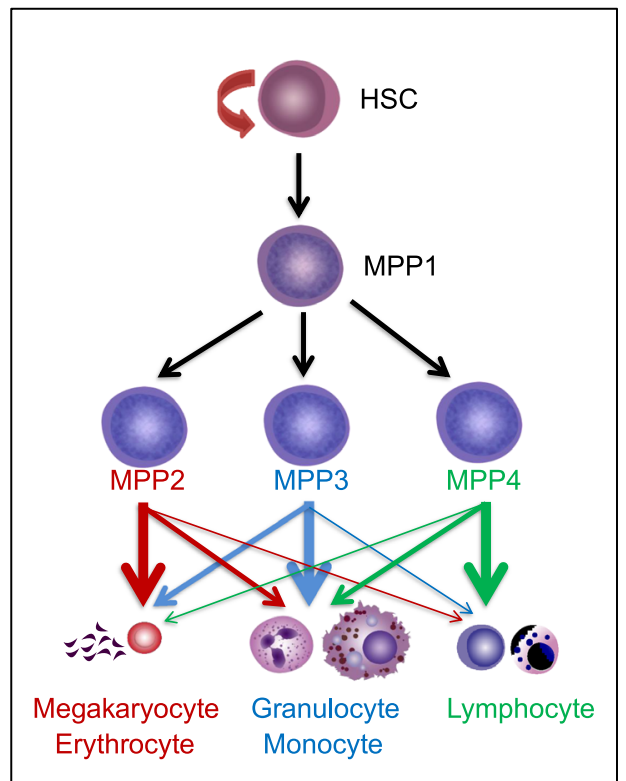

C

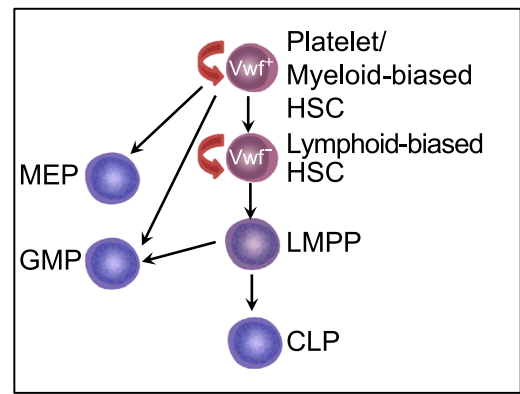

$\mathrm{F}$

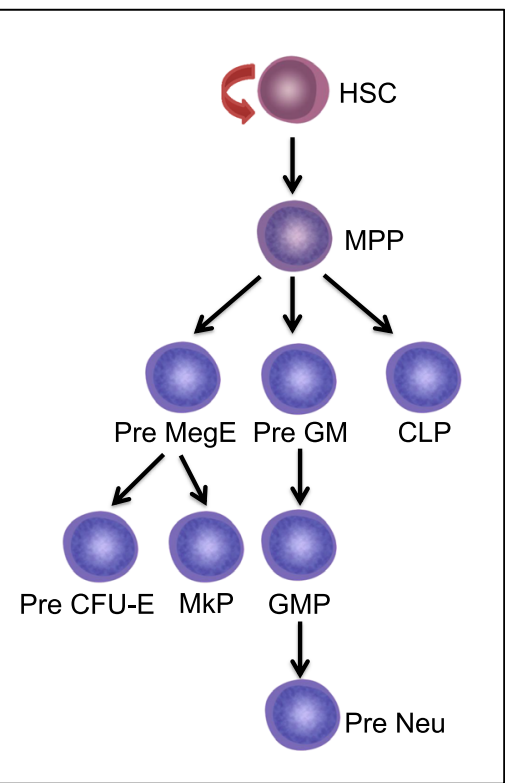

Figure 2. The revised models for hematopoietic stem cell differentiation. (A) My-Bi and Ly-Bi HSCs model. Ly-Bi HSCs reconstitute the myeloid lineage to a lesser extent than the lymphoid lineage, and vice versa. (B) Eaves' lab defined $\alpha, \beta, \gamma$, and $\delta$ cells according to the percentage of myeloid chimerism relative to that of lymphoid chimerism (M/L ratio). Single donor cell is defined as $\alpha$ cells when the $M / L$ ratio exceeds $2, \beta$ cells when $M / L$ ratio exceeds 0.25 but is less than 2 , and $\gamma / \delta$ cells when it is less than 0.25 . Therefore, $\alpha$ cells are myeloid-biased, $\beta$ cells are balanced, and $\gamma / \delta$ cells are lymphoid-biased without 2 nd transplantation capability. (C) $\mathrm{VWF}^{+}$platelet-biased HSCs sit at the apex of the hierarchy, and can differentiate into all progenitors and mature cells. VWF lymphoid-biased HSCs reside downstream of $\mathrm{VWF}^{+} \mathrm{HSCs}$. LMPPs cannot give rise to the megakaryocyte/erythrocyte lineage. MEPs are directly derived from HSCs. (D) In the myeloid bypass model, the LT-HSC population contains CMRPs, MERPs, and MkRPs. These MyRPs are directly produced by HSCs. (E) MPP subtypes are separated into MPP1-4. MPP1 can give rise to all lineages. MPP2/3 are myeloid-biased and MPP4 is lymphoid-biased. In addition, MPP2 is platelet-biased. (F) In this model, MPPs differentiate into pre MegE, Pre GM and CLP. Pre MegE is upstream of MkP and pre CFU-E. Pre GM gives rise to GMP, and subsequently generates newly defined neutrophil precursors (Pre Neu).

myeloid progenitors. In the LKS ${ }^{-}$population, $\mathrm{CD} 41^{+} \mathrm{CD} 150^{+}$ cells are defined as megakaryocyte progenitors (MkPs), which are exclusively associated with megakaryocyte generation. CD41-CD150- CD16/32+ cells are GMPs. In the CD41-CD150'CD16/32- population (classical CMPs and MEPs mixture), there are four newly defined sub-populations, including pre MegEs, pre GMs, Pre CFU-Es and CFUEs (Pronk et al., 2007). Single Pre MegE cells can effectively produce megakaryocytic, erythroid as well as mixed megakaryocyte/erythroid colonies. In contrast, Pre CFU-E cells give rise almost exclusively to erythroid colonies of various sizes. Pre GMs lie developmentally upstream of GMPs, and have a remarkably similar clonal lineage output to the GMPs. Therefore, the Pronk et al. study explores the processes of myeloid cell differentiation, reveals a number of novel intermediate progenitors, and orchestrates a new hierarchy model, including unipotent proliferative neutrophil precursors (Kim et al., 2017; Evrard et al., 2018; Zhu et al., 


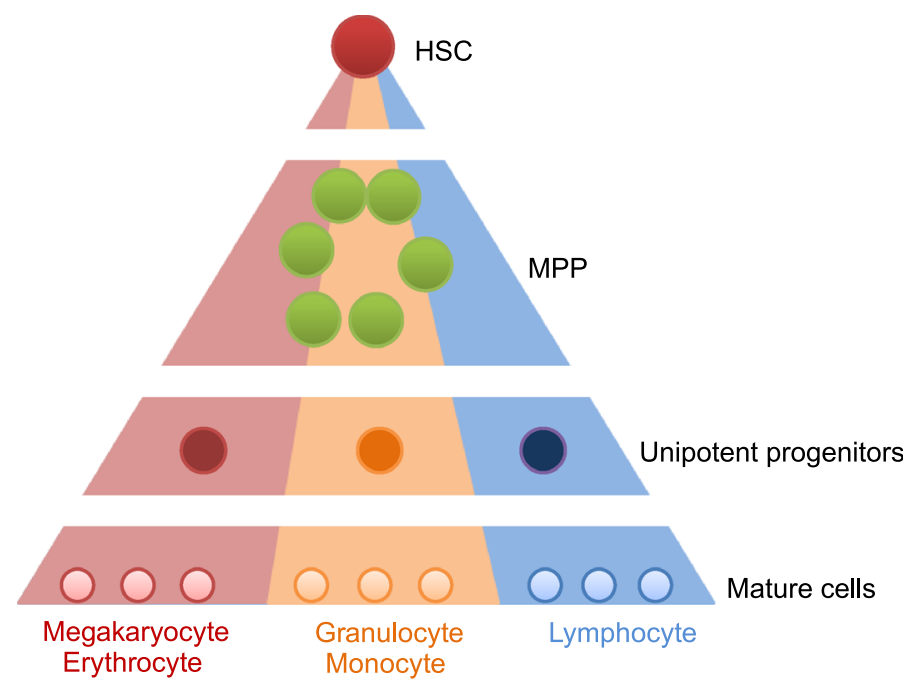

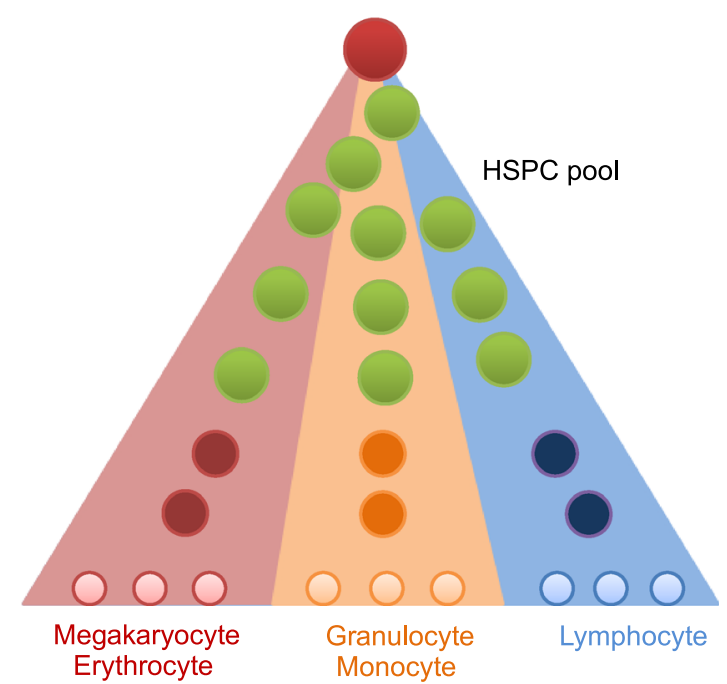

Figure 3. Discrete vs. continuous hematopoietic differentiation model. (A) The discrete differentiation model shows that HSCs differentiate to mature lineage the progression cells is a stepwise process following a tree-like hierarchy of oligo-, bi- and unipotent progenitors. (B) The continuous differentiation model shows that there is no obvious boundary in the hierarchy. Individual HSCs gradually acquire lineage biases along multiple directions without passing through discrete hierarchically organized progenitor populations.

2018). Classical CMPs consist of pre GMs, and the majority of pre MegEs and MEPs are separated into CFU-E, Pre CFU-Es, and part of pre MegEs. Moreover, MkPs are located mainly in CMPs (Pronk et al., 2007) (Fig. 2F).

In line with the work discussed above, a landmark paper from Amit's lab reporting the transcriptomes for more than 2,600 mouse single LKS $^{-}$myelo-erythroid progenitor cells (Paul et al., 2015) and a subsequent work from Gottgens' lab reporting the transcriptomes of 1,600 HSPCs (Nestorowa et al., 2016) both revealed heterogeneity in LKS progenitors. The single cells from classical MEPs do not show any expression of megakaryocyte markers or prominent megakaryocyte TFs. However, both megakaryocyte markers (Pf4 and CD41) and TFs (Pbx1, Fli1, Mef2c) are expressed in the cells from classical CMPs (Paul et al., 2015).

Taken together, all the studies explained why megakaryocytes mainly differentiate from CMPs, but not MEPs, and MEPs mostly give rise to erythrocytes. Therefore, this suggests that classical MEPs may not be the true precursor for megakaryocytes.

\section{Hematopoietic differentiation is a continuous process}

Previous studies indicated that individual HSCs gradually acquire lineage biases along multiple directions while passing through discrete hierarchically organized progenitor populations (Fig. 3A). However, these models are based on the analysis of predefined flow-sorted cell populations. With advances in methodologies, it has become possible to study the similarities or differences of individual HSPCs and their differentiation relationships.

By using scRNA-seq combined with computational analysis, a recent study on comprehensively sampled human bone marrow HSPCs suggested a model in which acquisition of lineage-specific fates is a continuous process, and unilineage restricted cells emerge directly from a continuum of low-primed undifferentiated HSPCs, without any major transition through the multi- and bi-potent stages (Velten et al., 2017). This view is supported by a zebrafish study that suggested that the continuum of HSPC differentiation is characterized by a highly coordinated transcriptional program, displaying simultaneous suppression of cell proliferation-related genes and upregulation of lineage specific genes (Macaulay et al., 2016). In addition, another study using human cord blood lympho-myeloid progenitor cells, including LMPPs, GMPs and multi-lymphoid progenitors (MLPs), further suggested a model in which a continuum of progenitors execute lymphoid and myeloid differentiation, rather than only unilineage progenitors being present downstream of stem cells (Karamitros et al., 2018). Although most progenitors have uni-lineage potential, bi- and oligolineage progenitors are present among LMPPs, GMPs and MLPs. These aforementioned studies change our view that hematopoietic differentiation is a continuous process, rather than a discrete hierarchy, which suggests that there is no obvious boundary among stem cells and progenitors (Laurenti and Gottgens, 2018) (Fig. 3B). 


\section{The roadmap of human hematopoiesis}

In human hematopoiesis, the Dick group sorted MPPs, CMPs and MEPs from fetal liver and adult bone marrow, and compared their lineage potential from different developmental stages (Notta et al., 2016). They showed that previously defined MPPs, CMPs and MEPs are heterogeneous. Importantly, MEPs, from both fetal liver and bone marrow, uniformly produce erythroid-only clones. Therefore, the classically defined MEPs are principally erythroid precursors when analyzed at single cell resolution and are not megakaryocyte/erythroid progenitors as previously thought, which is consistent with the observation in a mouse model (Pronk et al., 2007). Interestingly, fetal liver contains large numbers of distinct oligopotent progenitors. However, few oligopotent progenitors were present in the adult bone marrow. Instead only two progenitor classes predominate, multipotent and unipotent, with megakaryocyte/erythroid lineages emerging from multipotent cells. The Dick group's study provides a revised model to understand normal hematopoiesis, which is indeed flexible in developmental time.

\section{HEMATOPOIESIS UNDER PHYSIOLOGICAL CONDITION}

Hematopoiesis is regulated by microenvironment or niche (Morrison and Scadden, 2014; Crane et al., 2017), therefore, an unperturbed niche and a pretreated niche have different effects on hematopoiesis (Mendelson and Frenette, 2014). LT-HSC is considered to sit at the apex of the hierarchy, and reconstitutes or maintains the whole hematopoiesis. However, it is important to remember that almost all the supporting evidence was obtained from in vitro colony assay and in vivo transplantation. Irradiation or drug treatment can disrupt the niche, ablate the hematopoietic cells in the recipient, and create the space for donor HSPC engraftment, expansion and differentiation. Moreover, HSPCs are retained in the niche under hypoxic regulation (Suda et al., 2011; Nombela-Arrieta et al., 2013; Spencer et al., 2014; Itkin et al., 2016), and it is reported that transient exposure of HSPCs to normal oxygen impairs their function (Mantel et al., 2015). Therefore, transplantation of HSPCs into pretreated recipients cannot truly reflect the behaviors of hematopoiesis under physiological conditions (steady state).

To understand the dynamics of blood formation in steady state, various lineage-tracing approaches have been used to assess the lineage contribution of individual HSPCs in unperturbed hematopoiesis. By using a doxycycline-induced Sleeping Beauty transposon tagging approach in HSPCs, Sun et al. (Sun et al., 2014) reported that MPPs, rather than HSCs, are the main drivers of steady-state hematopoiesis during adulthood. In addition, Rodewald's lab devised a mouse model allowing inducible genetic labeling of the most primitive $\mathrm{Tie}^{+} \mathrm{HSCs}$ in bone marrow, and quantified label progression along hematopoietic development by limiting

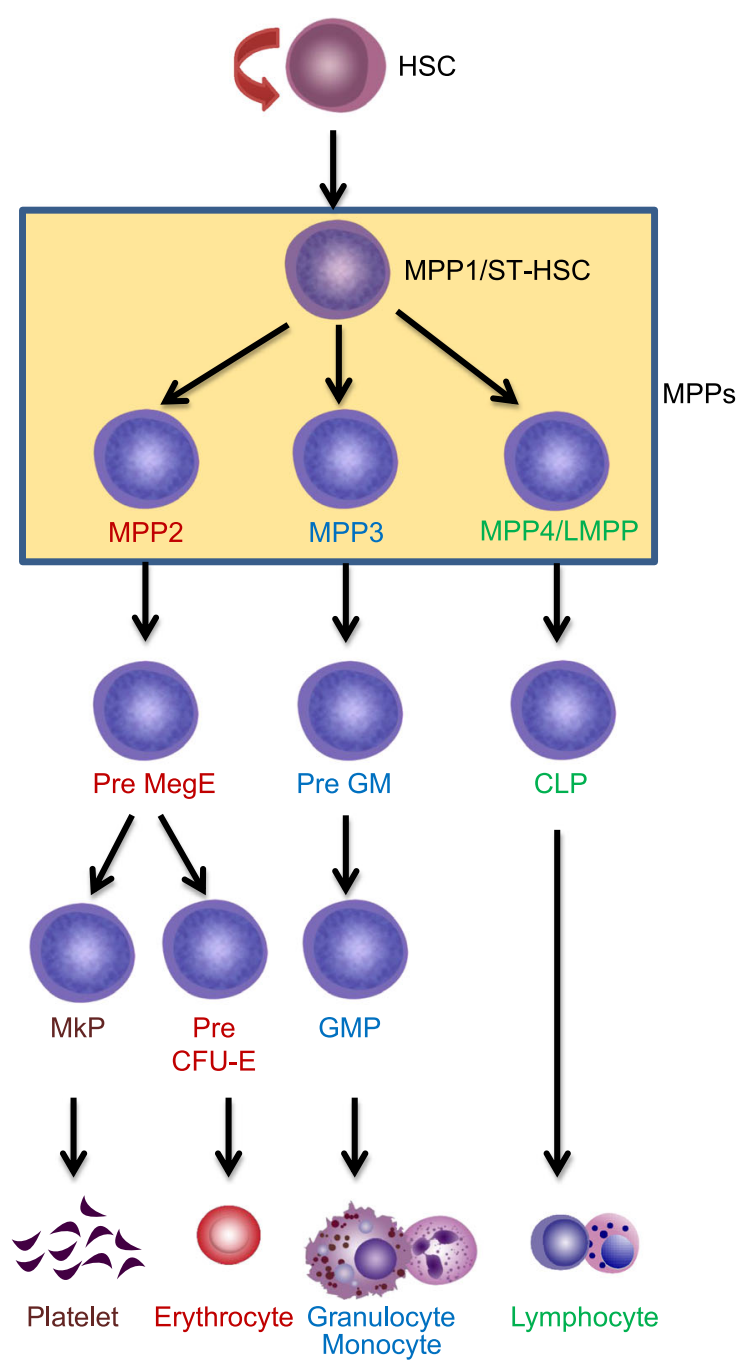

Figure 4. A reconciled model for hematopoietic stem cell differentiation. In this model, HSCs first differentiate into MPP1/ST-HSC, then give rise to MPP2, MPP3 and MPP4 (LMPP). MPP2 can generate pre MegE, and subsequently, pre MegE gives rise to platelets through MkP or produce erythrocytes through Pre CFU-E. MPP3 mostly give rise to granulocyte and monocyte lineages, and MPP4 (LMPP) mainly contribute to lymphocytes.

dilution analysis and mathematical modelling (Busch et al., 2015). They found that adult hematopoiesis is largely sustained by ST-HSC, and in contrast, LT-HSCs are rapidly used to establish the immune and blood system in fetal and early postnatal life. Another study from the same group used an alternative genetic fate-mapping system, called polylox barcoding (Pei et al., 2017), and demonstrated that when HSCs are labeled at the fetal liver stage, their descendants in the adult will mostly contribute to multiple lineages. However, when the analysis was repeated in adult stage, few barcodes were detected in HSCs as well as mature progenies. The above studies support the view that MPPs or STHSCs contribute predominantly to mature progenies, 
whereas HSCs do not have a notable role in steady-state hematopoiesis. However, Sawai et al. (2016) reported a paradoxical result. Pdzk1ip1 (Map17) is specifically expressed in the murine HSC population, therefore, they developed Pdzk1ip1-GFP and Pdzk1ip1-CreER; R26-TdTomato mice and showed that LT-HSCs provide a major contribution to all lineage-committed progenitors and mature blood cells. These controversial observations might be owing to the differences of tracing methods and labeling efficiency in HSCs. Therefore, new approaches should be developed and further investigation will be necessary to reconcile this paradox.

Interestingly, in Rodewald's polylox tracking study, they revealed a basic split between common myeloid-erythroid development and common lymphocyte development, supporting the bifurcating tree model of hematopoiesis that has not been proved in steady state (Pei et al., 2017). However, megakaryocytic fate was not analyzed in this study. Another study from the Camargo group addressed this issue more comprehensively (Rodriguez-Fraticelli et al., 2018). They performed a long-term (30-week) pulse-chase experiment in adult mice with the sleeping beauty barcode system, and found that during unperturbed hematopoiesis, the megakaryocyte lineage arises largely independently of other hematopoietic fates, and the LT-HSCs predominantly contribute to megakaryocyte output.

\section{CONCLUDING REMARKS}

Advanced technologies and new findings have broadened our knowledge on hematopoiesis. Hematopoietic hierarchy is more complicated than what we previously thought. In consideration of all the findings discussed above, the model shown in Fig. 4 represents the ideal hierarchy so far. However, we believe that with the advances in single cell technology, more subtypes of stem and progenitor cells will be discovered. Moreover, the epigenetic status of single HSPCs analyzed by single cell Hi-C (Nagano et al., 2013) and single cell ATAC-seq (Buenrostro et al., 2018; Cao et al., 2018; Cusanovich et al., 2018; Satpathy et al., 2018) will provide more information and the hierarchical model will be further revised. By combining imaging with in situ RNA-seq (Wang et al., 2018; Eng et al., 2019; Rodriques et al., 2019), studying the spatial localization of individual HSCs or HPCs becomes possible. This can help us know whether single HSPCs, with different transcriptomes, have specific lodgment sites in bone marrow.

The kinetics of adult HSC differentiation under different stress conditions have been studied recently in great detail (Lu et al., 2019). However, whether expansion of HSCs in recipients after transplantation occurs is still poorly understood. Furthermore, future studies can focus more on the changes of heterogeneity and hierarchy of HSPCs in disease. For example, one study reported that a $\mathrm{c}-\mathrm{Kit}^{\mathrm{hi}}$ progenitor subset positive for IL-7Ra emerged after infection of mice with Plasmodium chabaudi (Belyaev et al., 2010). These cells have both lymphoid and myeloid potential.
Therefore, studying HSPCs under different diseases is interesting and also important.

\section{ACKNOWLEDGMENTS}

We appreciate insightful suggestions from Dr. Hideo Ema. This work was supported by grants from the National Key Research and Development Program of China (2016YFA0100600, 2017YFA0 103400); the National Natural Science Foundation of China (81421002, 81730006, 81430004, 81670106, 81870086, 818110 1081); CAMS Initiative for Innovative Medicine (2017-I2M-3-009, 2016-I2M-1-017); and the CAMS Fundamental Research Funds for Central Research Institutes (2016GH3100001, 2018PT31005).

\section{ABBREVIATIONS}

$\mathrm{Ba}$, balanced; CLPs, common lymphoid progenitors; CMPs, common myeloid progenitors; CMRPs, common myeloid repopulating progenitors; FACS, fluorescence-activated cell sorting; GMPs, granulocyte-macrophage progenitors; HSCs, hematopoietic stem cells; HSPCs, hematopoietic stem and progenitor cells; IT-HSCs, intermediate-term hematopoietic stem cells; LKS', Lin ${ }^{-} \mathrm{CKit}^{+}$Sca1-; LMPPs, lymphoid-primed multipotent progenitors; LT-HSCs, long-term hematopoietic stem cells; Ly-Bi, lymphoid-biased; MEPs, megakaryocyte-erythrocyte progenitors; MERPs, megakaryocyte-erythrocyte repopulating progenitors; MkPs, megakaryocyte progenitors; MkRPs, megakaryocyte repopulating progenitors; MLPs, multi-lymphoid progenitors; MPPs, multipotent progenitors; My-Bi, myeloid-biased; scATAC-seq, single cell assay for transposase-accessible chromatin using sequencing; scRNA-seq, single-cell RNA sequencing; SLAM, signaling lymphocyte activation molecule; SL-MkPs, stem-like megakaryocyte committed progenitors; ST-HSC, short-term hematopoietic stem cells; TFs, transcription factors; vWF, von Willebrand factor.

\section{COMPLIANCE WITH ETHICS GUIDELINES}

Hui Cheng, Zhaofeng Zheng and Tao Cheng declare that they have no conflict of interest

\section{OPEN ACCESS}

This article is distributed under the terms of the Creative Commons Attribution 4.0 International License (http://creativecommons.org/ licenses/by/4.0/), which permits unrestricted use, distribution, and reproduction in any medium, provided you give appropriate credit to the original author(s) and the source, provide a link to the Creative Commons license, and indicate if changes were made.

\section{REFERENCES}

Adolfsson J, Mansson R, Buza-Vidas N, Hultquist A, Liuba K, Jensen CT, Bryder D, Yang L, Borge OJ, Thoren LA et al (2005) Identification of Flt3+ lympho-myeloid stem cells lacking erythromegakaryocytic potential a revised road map for adult blood lineage commitment. Cell 121:295-306 
Akashi K, Traver D, Miyamoto T, Weissman IL (2000) A clonogenic common myeloid progenitor that gives rise to all myeloid lineages. Nature 404:193-197

Belyaev NN, Brown DE, Diaz AI, Rae A, Jarra W, Thompson J, Langhorne J, Potocnik AJ (2010) Induction of an IL7-R(+)c-Kit(hi) myelolymphoid progenitor critically dependent on IFN-gamma signaling during acute malaria. Nat Immunol 11:477-485

Benveniste P, Frelin C, Janmohamed S, Barbara M, Herrington R, Hyam D, Iscove NN (2010) Intermediate-term hematopoietic stem cells with extended but time-limited reconstitution potential. Cell Stem Cell 6:48-58

Benz C, Copley MR, Kent DG, Wohrer S, Cortes A, Aghaeepour N, Ma E, Mader H, Rowe K, Day C et al (2012) Hematopoietic stem cell subtypes expand differentially during development and display distinct lymphopoietic programs. Cell Stem Cell 10:273283

Boyer SW, Schroeder AV, Smith-Berdan S, Forsberg EC (2011) All hematopoietic cells develop from hematopoietic stem cells through Flk2/FIt3-positive progenitor cells. Cell Stem Cell 9:6473

Bruns I, Lucas D, Pinho S, Ahmed J, Lambert MP, Kunisaki Y, Scheiermann C, Schiff L, Poncz M, Bergman A et al (2014) Megakaryocytes regulate hematopoietic stem cell quiescence through CXCL4 secretion. Nat Med 20(11):1315

Buenrostro JD, Corces MR, Lareau CA, Wu B, Schep AN, Aryee MJ, Majeti R, Chang HY, Greenleaf WJ (2018) Integrated single-cell analysis maps the continuous regulatory landscape of human hematopoietic differentiation. Cell 173:1535-1548 e1516

Busch K, Klapproth K, Barile M, Flossdorf M, Holland-Letz T, Schlenner SM, Reth M, Hofer T, Rodewald HR (2015) Fundamental properties of unperturbed haematopoiesis from stem cells in vivo. Nature 518:542-546

Cao J, Cusanovich DA, Ramani V, Aghamirzaie D, Pliner HA, Hill AJ, Daza RM, McFaline-Figueroa JL, Packer JS, Christiansen L et al (2018) Joint profiling of chromatin accessibility and gene expression in thousands of single cells. Science 361:1380-1385

Carrelha J, Meng Y, Kettyle LM, Luis TC, Norfo R, Alcolea V, Boukarabila H, Grasso F, Gambardella A, Grover A et al (2018) Hierarchically related lineage-restricted fates of multipotent haematopoietic stem cells. Nature 554:106-111

Crane GM, Jeffery E, Morrison SJ (2017) Adult haematopoietic stem cell niches. Nat Rev Immunol 17:573-590

Cusanovich DA, Hill AJ, Aghamirzaie D, Daza RM, Pliner HA, Berletch JB, Filippova GN, Huang X, Christiansen L, DeWitt WS et al (2018) A single-cell atlas of in vivo mammalian chromatin accessibility. Cell 174:1309-1324 e1318

Dick JE (2003) Stem cells: Self-renewal writ in blood. Nature 423:231-233

Dykstra B, Kent D, Bowie M, McCaffrey L, Hamilton M, Lyons K, Lee SJ, Brinkman R, Eaves C (2007) Long-term propagation of distinct hematopoietic differentiation programs in vivo. Cell Stem Cell 1:218-229

Eng CL, Lawson M, Zhu Q, Dries R, Koulena N, Takei Y, Yun J, Cronin C, Karp C, Yuan GC et al (2019) Transcriptome-scale super-resolved imaging in tissues by RNA seqFISH. Nature 568 (7751):235-239
Evrard M, Kwok IWH, Chong SZ, Teng KWW, Becht E, Chen J, Sieow JL, Penny HL, Ching GC, Devi S et al (2018) Developmental analysis of bone marrow neutrophils reveals populations specialized in expansion, trafficking, and effector functions. Immunity 48:364-379 e368

Forsberg EC, Serwold T, Kogan S, Weissman IL, Passegue E (2006) New evidence supporting megakaryocyte-erythrocyte potential of flk2/flt $3+$ multipotent hematopoietic progenitors. Cell 126:415-426

Haas S, Hansson J, Klimmeck D, Loeffler D, Velten L, Uckelmann H, Wurzer S, Prendergast AM, Schnell A, Hexel K et al (2015) Inflammation-induced emergency megakaryopoiesis driven by hematopoietic stem cell-like megakaryocyte progenitors. Cell Stem Cell 17(4):422-434

Huang $\mathrm{H}$, Cantor AB (2009) Common features of megakaryocytes and hematopoietic stem cells: what's the connection? J Cell Biochem 107:857-864

Ikuta K, Weissman IL (1992) Evidence that hematopoietic stem cells express mouse c-kit but do not depend on steel factor for their generation. Proc Natl Acad Sci USA 89:1502-1506

Itkin T, Gur-Cohen S, Spencer JA, Schajnovitz A, Ramasamy SK, Kusumbe AP, Ledergor G, Jung Y, Milo I, Poulos MG et al (2016) Distinct bone marrow blood vessels differentially regulate haematopoiesis. Nature 532:323-328

Jacobsen SEW, Nerlov C (2019) Haematopoiesis in the era of advanced single-cell technologies. Nat Cell Biol 21:2-8

Karamitros D, Stoilova B, Aboukhalil Z, Hamey F, Reinisch A, Samitsch M, Quek L, Otto G, Repapi E, Doondeea J et al (2018) Single-cell analysis reveals the continuum of human lymphomyeloid progenitor cells. Nat Immunol 19:85-97

Kiel MJ, Yilmaz OH, Iwashita T, Terhorst C, Morrison SJ (2005) SLAM family receptors distinguish hematopoietic stem and progenitor cells and reveal endothelial niches for stem cells. Cell 121:1109-1121

Kim MH, Yang D, Kim M, Kim SY, Kim D, Kang SJ (2017) A latelineage murine neutrophil precursor population exhibits dynamic changes during demand-adapted granulopoiesis. Sci Rep 7:39804

Kondo M, Weissman IL, Akashi K (1997) Identification of clonogenic common lymphoid progenitors in mouse bone marrow. Cell 91:661-672

Laurenti E, Gottgens B (2018) From haematopoietic stem cells to complex differentiation landscapes. Nature 553:418-426

Lu R, Czechowicz A, Seita J, Jiang D, Weissman IL (2019) Clonallevel lineage commitment pathways of hematopoietic stem cells in vivo. Proc Natl Acad Sci USA 116:1447-1456

Lu R, Neff NF, Quake SR, Weissman IL (2011) Tracking single hematopoietic stem cells in vivo using high-throughput sequencing in conjunction with viral genetic barcoding. Nat Biotechnol 29:928-933

Macaulay IC, Svensson V, Labalette C, Ferreira L, Hamey F, Voet T, Teichmann SA, Cvejic A (2016) Single-cell RNA-sequencing reveals a continuous spectrum of differentiation in hematopoietic cells. Cell Rep 14:966-977

Mantel CR, O'Leary HA, Chitteti BR, Huang X, Cooper S, Hangoc G, Brustovetsky N, Srour EF, Lee MR, Messina-Graham $S$ et al 
(2015) Enhancing hematopoietic stem cell transplantation efficacy by mitigating oxygen shock. Cell 161:1553-1565

Manz MG, Miyamoto T, Akashi K, Weissman IL (2002) Prospective isolation of human clonogenic common myeloid progenitors. Proc Natl Acad Sci USA 99:11872-11877

Mendelson A, Frenette PS (2014) Hematopoietic stem cell niche maintenance during homeostasis and regeneration. Nat Med 20:833-846

Metcalf D (2008) Hematopoietic cytokines. Blood 111:485-491

Moignard V, Macaulay IC, Swiers G, Buettner F, Schutte J, CaleroNieto FJ, Kinston S, Joshi A, Hannah R, Theis FJ et al (2013) Characterization of transcriptional networks in blood stem and progenitor cells using high-throughput single-cell gene expression analysis. Nat Cell Biol 15:363-372

Morita Y, Ema H, Nakauchi H (2010) Heterogeneity and hierarchy within the most primitive hematopoietic stem cell compartment. J Exp Med 207(6):1173-1182

Morrison SJ, Scadden DT (2014) The bone marrow niche for haematopoietic stem cells. Nature 505:327-334

Morrison SJ, Uchida N, Weissman IL (1995) The biology of hematopoietic stem cells. Annu Rev Cell Dev Biol 11:35-71

Morrison SJ, Wandycz AM, Hemmati HD, Wright DE, Weissman IL (1997) Identification of a lineage of multipotent hematopoietic progenitors. Development 124:1929-1939

Muller-Sieburg CE, Cho RH, Karlsson L, Huang JF, Sieburg HB (2004) Myeloid-biased hematopoietic stem cells have extensive self-renewal capacity but generate diminished lymphoid progeny with impaired IL-7 responsiveness. Blood 103:4111-4118

Muller-Sieburg CE, Cho RH, Thoman M, Adkins B, Sieburg HB (2002) Deterministic regulation of hematopoietic stem cell selfrenewal and differentiation. Blood 100:1302-1309

Nagano T, Lubling Y, Stevens TJ, Schoenfelder S, Yaffe E, Dean W, Laue ED, Tanay A, Fraser P (2013) Single-cell Hi-C reveals cellto-cell variability in chromosome structure. Nature 502:59-64

Nakorn TN, Miyamoto T, Weissman IL (2003) Characterization of mouse clonogenic megakaryocyte progenitors. Proc Natl Acad Sci USA 100:205-210

Nestorowa S, Hamey FK, Pijuan Sala B, Diamanti E, Shepherd M, Laurenti E, Wilson NK, Kent DG, Gottgens B (2016) A single-cell resolution map of mouse hematopoietic stem and progenitor cell differentiation. Blood 128:e20-31

Nombela-Arrieta C, Pivarnik G, Winkel B, Canty KJ, Harley B, Mahoney JE, Park SY, Lu J, Protopopov A, Silberstein LE (2013) Quantitative imaging of haematopoietic stem and progenitor cell localization and hypoxic status in the bone marrow microenvironment. Nat Cell Biol 15:533-543

Notta F, Zandi S, Takayama N, Dobson S, Gan OI, Wilson G, Kaufmann KB, McLeod J, Laurenti E, Dunant CF et al (2016) Distinct routes of lineage development reshape the human blood hierarchy across ontogeny. Science 351:aab2116

Oguro H, Ding L, Morrison SJ (2013) SLAM family markers resolve functionally distinct subpopulations of hematopoietic stem cells and multipotent progenitors. Cell Stem Cell 13:102-116

Okada S, Nakauchi H, Nagayoshi K, Nishikawa S, Miura Y, Suda T (1992) In vivo and in vitro stem cell function of c-kit- and Sca-1positive murine hematopoietic cells. Blood 80:3044-3050
Orkin SH (2000) Diversification of haematopoietic stem cells to specific lineages. Nat Rev Genet 1:57-64

Osawa M, Hanada K, Hamada H, Nakauchi H (1996) Long-term lymphohematopoietic reconstitution by a single CD34-low/negative hematopoietic stem cell. Science 273:242-245

Paul F, Arkin Y, Giladi A, Jaitin DA, Kenigsberg E, Keren-Shaul H, Winter D, Lara-Astiaso D, Gury M, Weiner A et al (2015) Transcriptional heterogeneity and lineage commitment in myeloid progenitors. Cell 163:1663-1677

Pei W, Feyerabend TB, Rossler J, Wang X, Postrach D, Busch K, Rode I, Klapproth K, Dietlein N, Quedenau C et al (2017) Polylox barcoding reveals haematopoietic stem cell fates realized in vivo. Nature 548:456-460

Pietras EM, Reynaud D, Kang YA, Carlin D, Calero-Nieto FJ, Leavitt $A D$, Stuart JM, Gottgens B, Passegue E (2015) Functionally distinct subsets of lineage-biased multipotent progenitors control blood production in normal and regenerative conditions. Cell Stem Cell 17:35-46

Pinho S, Marchand T, Yang E, Wei Q, Nerlov C, Frenette PS (2018) Lineage-biased hematopoietic stem cells are regulated by distinct niches. Dev Cell 44:634-641 e634

Pronk CJ, Rossi DJ, Mansson R, Attema JL, Norddahl GL, Chan CK, Sigvardsson M, Weissman IL, Bryder D (2007) Elucidation of the phenotypic, functional, and molecular topography of a myeloerythroid progenitor cell hierarchy. Cell Stem Cell 1:428-442

Reya T (2003) Regulation of hematopoietic stem cell self-renewal. Recent Progr Horm Res 58:283-295

Reya T, Morrison SJ, Clarke MF, Weissman IL (2001) Stem cells, cancer, and cancer stem cells. Nature 414:105-111

Robb L (2007) Cytokine receptors and hematopoietic differentiation. Oncogene 26:6715-6723

Rodriguez-Fraticelli AE, Wolock SL, Weinreb CS, Panero R, Patel $\mathrm{SH}$, Jankovic M, Sun J, Calogero RA, Klein AM, Camargo FD (2018) Clonal analysis of lineage fate in native haematopoiesis. Nature 553:212-216

Rodriques SG, Stickels RR, Goeva A, Martin CA, Murray E, Vanderburg CR, Welch J, Chen LM, Chen F, Macosko EZ (2019) Slide-seq: A scalable technology for measuring genomewide expression at high spatial resolution. Science 363:14631467

Sanjuan-Pla A, Macaulay IC, Jensen CT, Woll PS, Luis TC, Mead A, Moore S, Carella C, Matsuoka S, Jones TB et al (2013) Plateletbiased stem cells reside at the apex of the haematopoietic stemcell hierarchy. Nature 502(7470):232

Satpathy AT, Saligrama N, Buenrostro JD, Wei Y, Wu B, Rubin AJ, Granja JM, Lareau CA, Li R, Qi Y et al (2018) Transcript-indexed ATAC-seq for precision immune profiling. Nat Med 24:580-590

Sawai CM, Babovic S, Upadhaya S, Knapp D, Lavin Y, Lau CM, Goloborodko A, Feng J, Fujisaki J, Ding L et al (2016) Hematopoietic stem cells are the major source of multilineage hematopoiesis in adult animals. Immunity 45:597-609

Seita J, Weissman IL (2010) Hematopoietic stem cell: self-renewal versus differentiation. Wiley Interdiscip Rev Syst Biol Med 2:640653

Shin JY, Hu W, Naramura M, Park CY (2014) High c-Kit expression identifies hematopoietic stem cells with impaired self-renewal and megakaryocytic bias. J Exp Med 211:217-231 
Spangrude GJ, Heimfeld S, Weissman IL (1988) Purification and characterization of mouse hematopoietic stem cells. Science 241:58-62

Spencer JA, Ferraro F, Roussakis E, Klein A, Wu J, Runnels JM, Zaher W, Mortensen LJ, Alt C, Turcotte R et al (2014) Direct measurement of local oxygen concentration in the bone marrow of live animals. Nature 508:269-273

Suda T, Takubo K, Semenza GL (2011) Metabolic regulation of hematopoietic stem cells in the hypoxic niche. Cell Stem Cell 9:298-310

Sugiyama T, Kohara H, Noda M, Nagasawa T (2006) Maintenance of the hematopoietic stem cell pool by CXCL12-CXCR49 chemokine signaling in bone marrow stromal cell niches. Immunity 25:977-988

Sun J, Ramos A, Chapman B, Johnnidis JB, Le L, Ho YJ, Klein A, Hofmann O, Camargo FD (2014) Clonal dynamics of native haematopoiesis. Nature 514:322-327

Till JE, Mc CE (1961) A direct measurement of the radiation sensitivity of normal mouse bone marrow cells. Radiat Res 14:213-222

Velten L, Haas SF, Raffel S, Blaszkiewicz S, Islam S, Hennig BP, Hirche C, Lutz C, Buss EC, Nowak D et al (2017) Human haematopoietic stem cell lineage commitment is a continuous process. Nat Cell Biol 19:271-281

Wang JF, Liu ZY, Groopman JE (1998) The alpha-chemokine receptor CXCR74 is expressed on the megakaryocytic lineage from progenitor to platelets and modulates migration and adhesion. Blood 92:756-764

Wang X, Allen WE, Wright MA, Sylwestrak EL, Samusik N, Vesuna S, Evans K, Liu C, Ramakrishnan C, Liu J et al (2018) Threedimensional intact-tissue sequencing of single-cell transcriptional states. Science 361(6400):eaat5691

Wilson A, Laurenti E, Oser G, van der Wath RC, Blanco-Bose W, Jaworski M, Offner S, Dunant CF, Eshkind L, Bockamp E et al (2008) Hematopoietic stem cells reversibly switch from dormancy to self-renewal during homeostasis and repair. Cell 135:11181129

Wilson NK, Kent DG, Buettner F, Shehata M, Macaulay IC, CaleroNieto FJ, Sanchez Castillo M, Oedekoven CA, Diamanti E, Schulte $R$ et al (2015) Combined single-cell functional and gene expression analysis resolves heterogeneity within stem cell populations. Cell Stem Cell 16:712-724

Yamamoto R, Morita Y, Ooehara J, Hamanaka S, Onodera M, Rudolph KL, Ema H, Nakauchi H (2013) Clonal analysis unveils self-renewing lineage-restricted progenitors generated directly from hematopoietic stem cells. Cell 154:1112-1126

Yang L, Bryder D, Adolfsson J, Nygren J, Mansson R, Sigvardsson M, Jacobsen SE (2005) Identification of Lin(-)Sca1(+)kit(+)CD34 $(+)$ Flt3- short-term hematopoietic stem cells capable of rapidly reconstituting and rescuing myeloablated transplant recipients. Blood 105:2717-2723

Yoshihara H, Arai F, Hosokawa K, Hagiwara T, Takubo K, Nakamura Y, Gomei Y, Iwasaki H, Matsuoka S, Miyamoto K et al (2007) Thrombopoietin/MPL signaling regulates hematopoietic stem cell quiescence and interaction with the osteoblastic niche. Cell Stem Cell 1:685-697

Zhang CC, Lodish HF (2008) Cytokines regulating hematopoietic stem cell function. Curr Opin Hematol 15:307-311

Zhao M, Perry JM, Marshall H, Venkatraman A, Qian P, He XC, Ahamed J, Li L (2014) Megakaryocytes maintain homeostatic quiescence and promote post-injury regeneration of hematopoietic stem cells. Nat Med 20(11):1321

Zhu J, Emerson SG (2002) Hematopoietic cytokines, transcription factors and lineage commitment. Oncogene 21:3295-3313

Zhu YP, Padgett L, Dinh HQ, Marcovecchio P, Blatchley A, Wu R, Ehinger E, Kim C, Mikulski Z, Seumois G et al (2018) Identification of an early unipotent neutrophil progenitor with pro-tumoral activity in mouse and human bone marrow. Cell Rep 24:23292341 e2328 